\title{
Using Multisensory Technologies to Stimulate People: a Reflexive Paper on Scenagrams
}

\author{
Céline Jost
}

Paris 8 University, CHArt laboratory, celine.jost@univ-paris8.fr

Brigitte Le Pévédic

South Brittany, Lab-STICC laboratory, brigitte.le-pevedic@univ-ubs.fr

Gérard Uzan

Paris 8 University, CHArt laboratory, Gerard.uzan@univ-ubs.fr

This paper aims at discussing the interest to use multisensory technologies for humans cognition training. First it introduces multisensory interactions making a focus on advancement in two fields: Human-Computer Interaction and mulsemedia. Second, it presents two different multisensory systems resulting from Robadom and StimSense projects that could be adapted for the community. Then, this paper defines the concept of scenagram and gives its application scopes, boundaries and use cases, offering a first classification of this new concept.

CCS CONCEPTS • Human-centered computing • Applied Computing • Software and its engineering

Additional Keywords and Phrases: multisensory interaction, multisensory systems, cognition, user experience, use cases, scenagram

ACM Reference Format:

Céline Jost, Brigitte Le Pévédic and Gérard Uzan. Using Multisensory Technologies to Stimulate People: a Reflexive Paper on Scenagrams. In SensoryX '21: Workshop on Multisensory Experiences, together with IMX 2021: ACM International Conference on Interactive Media Experiences. June 21-23, 2021. NY, USA.

\section{INTRODUCTION}

In the field of Human-Computer Interaction $(\mathrm{HCl})$, and with the development of Natural User interfaces, researchers are more and more studying multisensory interaction because this is the natural way for humans to communicate [1][2]. Indeed, humans evolve in a multisensory environment. The brain is naturally made to manage sensory inputs. With the emergence of technologies, systems can now integrate sensory devices allowing reproducing natural communication, making humans more cognitively efficient than with classical systems [3][4][5]. But, as for all artificial systems, we need to understand how the natural processes work to be able to reproduce them. The main difficulty with multisensory interaction is its direct impact on the brain since senses are part of humans. In a relatively new literature review, we concluded that there are, indeed, some

Published in accordance with the terms of the Creative Commons Attribution 4.0 International Public License (CC BY 4.0). Permission to reproduce or distribute this work, in part or in whole, verbatim, adapted, or remixed, is granted without fee, provided that the appropriate credits are given to the original work, not implying any endorsement by the authors or by SBC.

2021 Brazilian Computing Society 
really good user experiences thanks to technological multisensory interaction, but also some really bad experiences [6]. And more and more researchers from different disciplines are currently focusing on two main issues. First, they aim at providing computing architectures that can manage and synchronize sensory devices between them and between, for example, audiovisual content in the case of mulsemedia [7][8]. Second, they aim at studying the impacts of sensory effects (wind, vibration, water, scent, light, ...) and groups of effects on humans to improve cognition, to increase the quality of user experience, and/or to provide systems designed for all. Indeed, in a multisensory interactive system, the user can interact through several modalities for the same objective, thus the system automatically adapt to user according to her/his capabilities or preferences. We observed that mulsemedia research might be the most advanced on the topic of multisensory stimulation [9][10][11] and that $\mathrm{HCl}$ research might be the most advanced on the topic of interactions. Actually, we observed that each discipline seemed to take interest in different parts of the issue and that each discipline can therefore enrich the others. Since our research deals with multisensory interaction, which seems to be the intersection between $\mathrm{HCl}$ and mulsemedia, the objective of our paper is to initiate collaborations between $\mathrm{HCl}$ and mulsemedia researchers by presenting what we have achieved so far, in the field of $\mathrm{HCl}$, and our thoughts about the multisensory technologies which are specifically conceived for cognition training (in which we include mulsemedia). Section 2 presents two systems we built to allow end-users to create multimodal programs that we would like to discuss on. And Section 3 presents the concept of scenagram, focusing on application scopes, boundaries and use cases ; it also details our first thoughts about some possible ways of using systems that work on cognition (stimulation or learning).

\section{ARCO AND MULSEBOX: TWO MULTISENSORY SYSTEMS}

We were first interested in multisensory interaction for its capability to reduce cognitive decline [12][13]. Indeed, it can help recall memories, correct and improve literacy skills or improve selective attention [3][4]. More generally, it is helpful for all learning processes. Our objective was therefore to provide a system that can offer multisensory cognitive stimulation exercises. For this purpose, we investigated two different approaches to solve this issue: using the existing environment (all the devices owned by the end-user) or creating an independent multisensory device.

The first approach was investigated in the Robadom project [14] where we developed ArCo [15], a computing architecture that makes devices compatible to communicate together using a Java server. Generally, a device can send information about the environment (called perception) and/or can make actions on the environment (called action). To be compatible with ArCo, a device has to respond to the following requirements. First, it must provide a small XML description of its perceptions and actions. Second, for each device, developers have to provide a short Java program which makes the link between XML instructions and the real perception/action from/to the associated device. A library allows to easily integrate devices to the architecture, which is made to be very simple to use, without a time-consuming learning. A visual programming language interface (AmbiProg) allows users to create some applications using all these devices (see Figure 1). AmbiProg displays the list of all the known perceptions and actions and allows the user to use them within algorithms. Thus, it is really easy and fast to create a scenario using users' devices. The advantage of such a system is to be easily integrated into an existing environment and to offer immersive and spatialized applications with devices settled all around the user in the whole room. It's not built for mulsemedia and does not provide tools such as SEMP or PlaySEM [16]. And it would be very interesting to make them collaborate. For example, if PlaySEM could be able to send 
perceptions during the movie playback, these perceptions could be used by algorithms designed with AmbiProg, which would allow to take humans actions into account during the mulsemedia.

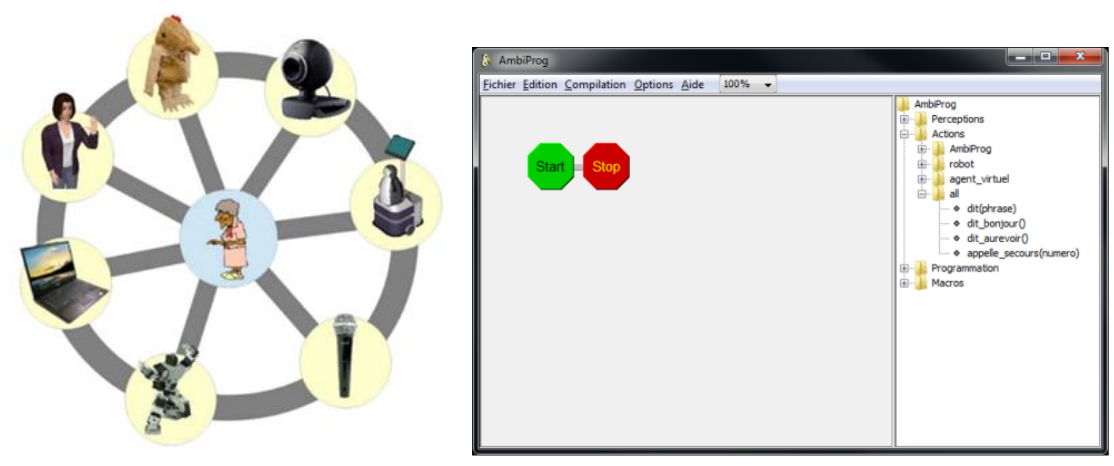

Figure 1: On the left, an illustration of ArCo concept. On the right, a screenshot of AmbiProg.

The second approach was investigated in the StimSense project [6] where we developed MulseBox, a portable multisensory interactive device (see Figure 2). It is limited in terms of perceptions and actions. About perceptions, it contains 2 ultrasounds, a RFID reader, 4 buttons. About actions, it contains 2 vibrators, 6 diods, a fan, a screen, and 2 speakers.The advantage of such a system is to be easily moved and used (and demonstrated) in different places, for example, in homecare centers or retirement homes. It is also interesting to have a common tool to share applications between researchers and to study the impact of sensory effects.

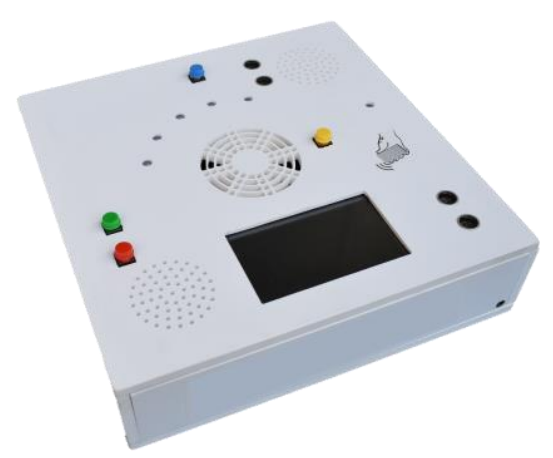

Figure 2 : MulseBox: our portable multisensory interactive device prototype

\section{APPLICATION SCOPES, BOUNDARIES, AND USE CASES}

Our first motivation was to build a system able to play cognitive stimulation exercises based on Activities of Daily Living, which are known to increase neuropsychological performances [17]. Indeed, to improve cognition, exercises must be relevant for patients and ensure the transfer of learning, which is possible only between exercises that require the same cognitive functions. For example, clicking on a virtual button with a mouse does not require the same processes as clicking on a real button with one's finger (engaging much more muscles). 
Therefore each patient should practice exercises that can be applied to her/his own life [18] and that are done with daily life objects [19].

With time, we understood that multisensory interaction had the potential to reproduce real-life, allowing creating an infinity of activities in controlled ecology. In our literature review [6], we draw up a list of at least 8 disciplines currently investigating multisensory interaction and at least 18 possibles application scopes: cinema, telecommunication, education, telemedicine, health, commerce, advertising/marketing, gaming/entertainment, multimedia, art, storytelling, museum, attractions, theater, product design, virtual reality, music, and home. We can also add driving [20].

The question was, therefore: what for? What can we or should we do with an artificial multisensory interaction? Keeping in mind our need to create real activities in controlled ecology to stimulate cognition, we tried to make a first classification of possible and useful types of activities made to stimulate users. In this context, we consider the interaction as a triad: trainer, learner, and goal (knowledge). And we define the concept of scenagram which is: a series of actions done by the end-user and/or by digital devices, alternately, to reach a common goal based on cognitive stimulation.

Thus, in our context, scenagrams aim at reproducing a learning situation between a trainer and a learner (for example improving memory with cognitive stimulation exercises). The system plays the role of the trainer, being a guide or a coach, which can assist the learner simulating daily-life activities, role plays, serious games, problem solving, case studies, and other interaction scenarios.

Table 1 presents what scenagrams are. We divided them into two different categories based on two modes: an autonomous mode where the system plays preconfigured actions and an interactive mode where users must be involved in the interaction.

In the autonomous mode, users can only start, pause, or stop the interaction. Despite this weak participation of users, this mode can be used to design an infinity of scenagrams where users must watch the system. In addition to mulsemedia, one could design some demonstrations (like demonstration mode in shops), some procedures like tutorials, training courses, or cooking recipes, some situations where users must "read" a user manual or to "listen" to a story. In a multisensory world, reading and listening requires all our senses. Lights, vibrations, motions, and so on can also come along.

In the interactive mode, the interaction is more complex. One can design all kinds of Questions and Answers exercises knowing that a question is multisensorial and can be represented by choreographies of lights, images, sounds, motions, and other sensory effects; and so does the answer. It opens the possibility to design some imitation games (useful for imitative learning), synchronization games (for example to learn music rhythm), or reaction games. It is also possible to design some more complex scenagrams, for example, interactive guidance where users practice taking the bus, going to the supermarket, and paying at the grocery store. It is also possible to create some memory games with sequences that must be reproduced, such as adaptations of Simon's game. Moreover, all kinds of quizzes can be designed. Last, this mode comes in addition to the autonomous mode with more complex procedures such as setting the oven time, scheduling a movie recording, writing an SMS, learning how to identify the reason of a breakdown, and so on.

Table 1: Classification of multisensory interaction situations for cognition constituting scenagrams

\begin{tabular}{ll}
\hline Autonomous mode with possible pauses/step-by-step & Questions and Answers mode/exercises \\
\hline Mulsemedia & Imitation/reproduction \\
Demonstration & Synchronization
\end{tabular}




\begin{tabular}{ll}
\hline Autonomous mode with possible pauses/step-by-step & Questions and Answers mode/exercises \\
\hline Procedure (tutorial, training course, recipe, ...) & Reaction games \\
Manual reading & Interactive guidance/story \\
Story listening & Memory game \\
& Quiz \\
& Procedure
\end{tabular}

\section{CONCLUSION}

In this paper, we presented ArCo and MulseBox two multisensory systems we could provide to the community in order to have a common background to study sensory effects. Moreover, this paper also presented the notion of scenagram along with a first classification of possible use cases for such interactive learning situations systems. We could later enrich it with the community to establish more complete specifications of scenagrams.

\section{ACKNOWLEDGMENTS}

We would like to thank all the people who have participated in the Robadom and StimSense projects and who have helped us to develop our thoughts as well as their partners. And we would also like to thank our institutions who give us invaluable support all year round.

\section{REFERENCES}

[1] A. Dix, "Human-computer interaction, foundations and new paradigms," J. Vis. Lang. Comput., vol. 42, pp. 122-134, Oct. 2017.

[2] G. Goth and Gregory, "Brave NUI world," Commun. ACM, vol. 54, no. 12, p. 14, Dec. 2011.

[3] R. Casillas Figueroa, "Personal spaces for multisensory stimulation as support to rehabilitate patients with cognitive disabilities," in Proceedings of the XVIII International Conference on Human Computer Interaction - Interacción '17, 2017, pp. 1-2.

[4] A. Covaci, L. Zou, I. Tal, G.-M. Muntean, and G. Ghinea, "Is Multimedia Multisensorial? - A Review of Mulsemedia Systems," ACM Comput. Surv., vol. 51, no. 5, pp. 1-35, 2018.

[5] Y. Sulema, "Mulsemedia vs. Multimedia: State of the art and future trends," in 2016 International Conference on Systems, Signals and Image Processing (IWSSIP), 2016, pp. 1-5.

[6] C. Jost, B. Le Pevedic, O. El Barraj, and G. Uzan, "MulseBox: Portable multisensory interactive device," in Conference Proceedings IEEE International Conference on Systems, Man and Cybernetics, 2019, vol. 2019-Octob, pp. 3956-3961.

[7] E. B. Saleme, C. A. S. Santos, and G. Ghinea, "A mulsemedia framework for delivering sensory effects to heterogeneous systems," Multimed. Syst., vol. 25, no. 4, pp. 421-447, Aug. 2019.

[8] E. B. Saleme, A. Covaci, G. Mesfin, C. A. S. Santos, and G. Ghinea, "Mulsemedia DIY: A survey of devices and a tutorial for building your own mulsemedia environment," ACM Computing Surveys, vol. 52, no. 3. Association for Computing Machinery, 01-Jun-2019.

[9] K. Brunnström et al., "Qualinet White Paper on Definitions of Quality of Experience," 2013.

[10] A. Perkis, C. Timmerer, and et al., "QUALINET White Paper on Definitions of Immersive Media Experience (IMEx)," in European Network on Quality of Experience in Multimedia Systems and Services, 14th QUALINET meeting (online), 2019.

[11] N. Murray, O. A. Ademoye, G. Ghinea, and G. M. Muntean, "A tutorial for olfaction-based multisensorial media application design and evaluation," ACM Comput. Surv., vol. 50, no. 5, Sep. 2017.

[12] S. Baillon, E. van Diepen, and R. Prettyman, "Multi-sensory therapy in psychiatric care," Adv. Psychiatr. Treat., vol. 8, no. 6, pp. 444450, Nov. 2002.

[13] J. C. Chung and C. K. Lai, "Snoezelen for dementia," Cochrane Database Syst. Rev., no. 4, Oct. 2002. 
[14] M. Chetouani et al., "Cognitive services for elderly people: The ROBADOM project," 2010.

[15] C. Jost, B. Le Pévédic, and D. Duhaut, "ArCo: an architecture for children to control a set of robots."

[16] E. B. Saleme and C. A. S. Santos, "PlaySEM: a Platform for Rendering MulSeMedia Compatible with MPEG-V," in Proceedings of the 21st Brazilian Symposium on Multimedia and the Web - WebMedia '15, 2015, pp. 145-148.

[17] S. T. Farias, E. Harrell, C. Neumann, and A. Houtz, "The relationship between neuropsychological performance and daily functioning in individuals with Alzheimer's disease: ecological validity of neuropsychological tests," Arch. Clin. Neuropsychol., vol. 18, no. 6, pp. 655672, Aug. 2003.

[18] V. Wojtasik, F. Lekeu, A. Quittre, C. Olivier, S. Adam, and É. Salmon, "Réadaptation cognitive pour les activités de vie quotidiennedans la maladie d'Alzheimer," Gérontologie et société, vol. 32 / n 130, no. 3, p. 187, 2009.

[19] B. Bouchard, F. Imbeault, A. Bouzouane, and B.-A. J. Menelas, "Developing Serious Games Specifically Adapted to People Suffering from Alzheimer," Springer, Berlin, Heidelberg, 2012, pp. 243-254.

[20] D. Dmitrenko, E. Maggioni, and M. Obrist, "Towards a framework for validating the matching between notifications and scents in olfactory in-car interaction," in Conference on Human Factors in Computing Systems - Proceedings, 2019. 